\title{
Clinical Indications for Newer Antifungal Agents
}

\author{
Nina Naeger-Murphy, Pharmo ${ }^{1,2}$ \\ James C. Pile, $\mathrm{MD}^{1,3}$ \\ ${ }^{1}$ Division of Infectious Diseases, Case Western \\ Reserve University at MetroHealth Medical \\ Center, Cleveland, Ohio \\ ${ }^{2}$ Department of Pharmacy, Case Western Re- \\ serve University at MetroHealth Medical Center, \\ Cleveland, Ohio \\ ${ }^{3}$ Division of Hospital Medicine, Case Western \\ Reserve University at MetroHealth Medical Cen- \\ ter, Cleveland, Ohio
}

\begin{abstract}
Recent years have seen the release of multiple new systemic antifungal agents, significantly increasing options for the treatment of most serious fungal infections. Newly available drugs include those in the echinocandin class, including caspofungin, micafungin, and anidulafungin, as well as the newer generation triazoles, voriconazole and posaconazole. Ordering of these agents is variably restricted, depending on a given institution's policies, and all are costly. In this review we examine the available evidence and outline the role of newer antifungal medications in several common and/or important situations, including invasive and mucocutaneous Candida infection, febrile neutropenia, invasive aspergillosis, zygomycosis, and endemic mycoses. Journal of Hospital Medicine 2008;4:102-111. 두 2009 Society of Hospital Medicine.
\end{abstract}

KEYWORDS: antifungal drugs, Aspergillus, candidemia, febrile neutropenia, fungal infection.

$\mathbf{T}$ herapy of serious fungal infections, for decades largely limited to the deoxycholate ("regular") preparation of amphotericin B (D-AmB), expanded significantly with the introduction of fluconazole, followed by lipid-based formulations of amphotericin B (L$\mathrm{AmB}$ ) and itraconazole. More recently the antifungal armamentarium has broadened further with the approval of voriconazole and posaconazole, as well as the echinocandins caspofungin, micafungin, and anidulafungin. Clinicians, including hospitalists, primary care, emergency medicine, and critical care physicians, may find it challenging to remain abreast of indications for these novel agents, and we review these below, with a focus on adult patients. Manuscripts used in the review were identified by a search of Englishlanguage articles in the PubMed MEDLINE database from 1994 to the present, using the keywords "triazoles," "echinocandins," "voriconazole,” "posaconazole,” “caspofungin,” "micafungin,” “anidulafungin," “candidemia," “candidiasis,” "aspergillosis," "invasive Aspergillus," “zygomycosis," "febrile neutropenia," "endemic mycosis," "histoplasmosis," and "coccidioidomycosis." In addition, reference lists for the majority of the identified manuscripts were hand-searched for additional pertinent citations.

Table 1 summarizes the newer systemic antifungal therapies and Table 2 summarizes the significant drug-drug interactions with the newer antifungals.

\section{INVASIVE CANDIDIASIS}

Candida has become a leading cause of nosocomial bloodstream infections, and is associated with an attributable mortality of $15 \%$ to $25 \%{ }^{1}$ Candidemia results in an estimated 10-day increase in hospital length of stay, as well as an average $\$ 40,000$ (US) increase in costs. ${ }^{2}$ Invasive candidiasis may be defined as 


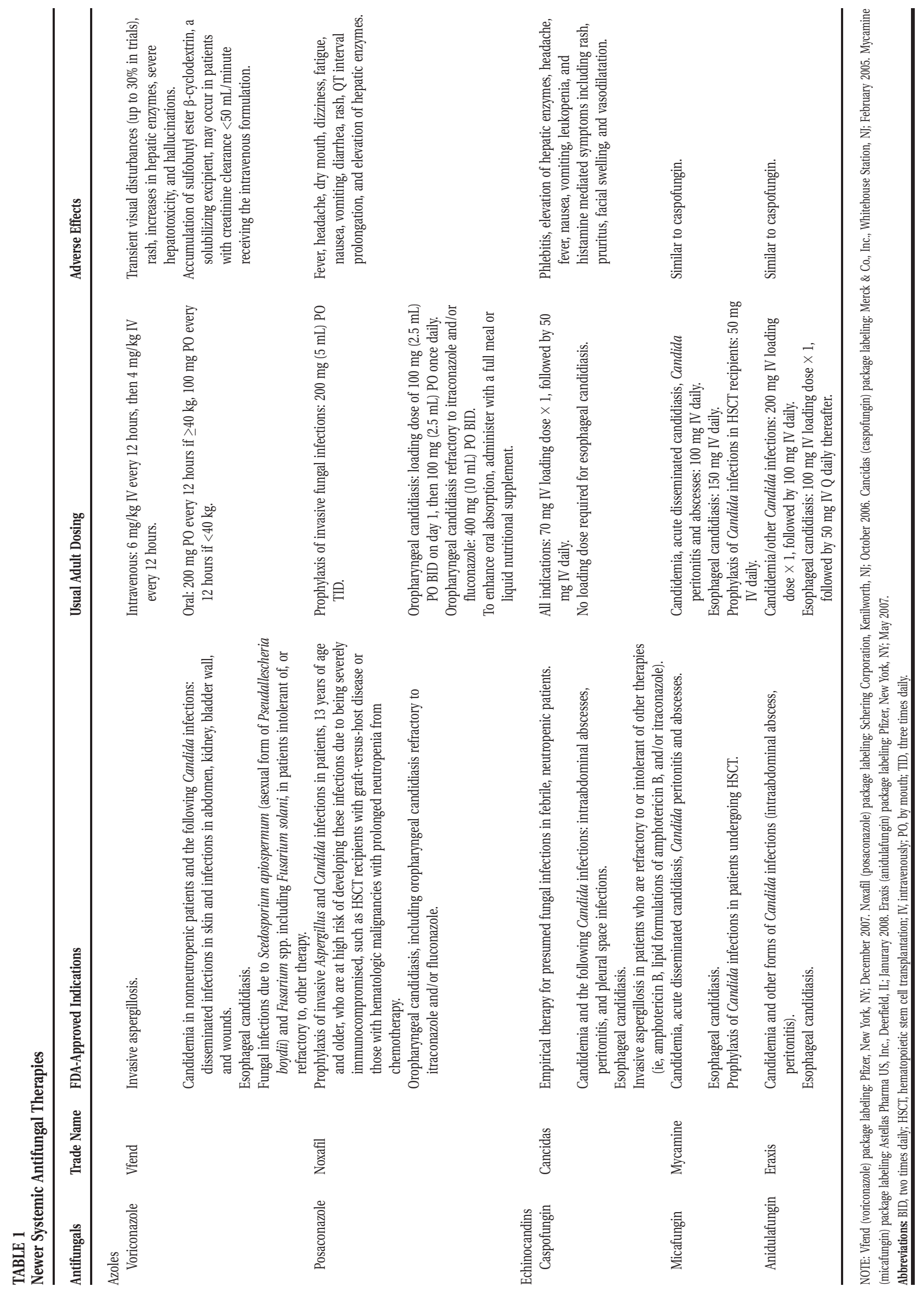


TABLE 2

Significant Drug-Drug Interactions with the Newer Antifungals

\begin{tabular}{|c|c|c|}
\hline Antifungal & Effect & Interacting Drugs \\
\hline \multirow[t]{3}{*}{ Voriconazole } & Decreased azole serum concentration & $\begin{array}{l}\text { Rifampin, rifabutin, carbamazepine, long-acting barbiturates, efavirenz, high-dose ritonavir } \\
\text { (400 mg twice daily), phenytoin }\end{array}$ \\
\hline & Increased azole serum concentration & $\begin{array}{l}\text { Oral contraceptives containing ethinyl estradiol and norethindrone, HIV protease inhibitors } \\
\text { other than ritonavir, and nonnucleoside reverse transcriptase inhibitors other than efavirenz }\end{array}$ \\
\hline & $\begin{array}{l}\text { Increased serum concentration of } \\
\text { coadministered drug }\end{array}$ & $\begin{array}{l}\text { Sirolimus, rifabutin, efavirenz, terfenadine, astemizole, cisapride, pimozide, quinine, cyclosporine, } \\
\text { methadone, tacrolimus, oral contraceptives containing ethinyl estradiol and norethindrone, HIV } \\
\text { protease inhibitors other than ritonavir, nonnucleoside reverse transcriptase inhibitors other than } \\
\text { efavirenz, benzodiazepines, HMG-CoA reductase inhibitors, dihydropyridine calcium channel } \\
\text { blockers, vinca alkaloids, omeprazole, phenytoin, warfarin, sulfonylurea oral hypoglycemics, and } \\
\text { ergot alkaloids }\end{array}$ \\
\hline \multirow[t]{2}{*}{ Posaconazole } & Decreased azole serum concentration & Cimetidine, rifabutin, phenytoin \\
\hline & $\begin{array}{l}\text { Increased serum concentration of } \\
\text { coadministered drug }\end{array}$ & $\begin{array}{l}\text { Cyclosporine, tacrolimus, rifabutin, midazolam, pheytoin, terfenidine, astemizole, pimozide, cisapride, } \\
\text { quinidine, ergot alkaloids, vinca alkaloids, sirolimus, HMG Co-A reductase inhibitors, and calcium } \\
\text { channel blockers }\end{array}$ \\
\hline \multirow[t]{3}{*}{ Caspofungin } & $\begin{array}{l}\text { Decreased serum concentration of } \\
\text { caspofungin }\end{array}$ & Efavirenz, nevirapine, phenytoin, dexamethasone, and carbamazepine \\
\hline & $\begin{array}{l}\text { Increased serum concentration of } \\
\text { caspofungin }\end{array}$ & Cyclosporine \\
\hline & $\begin{array}{l}\text { Decreased serum concentration of } \\
\text { coadministered drug }\end{array}$ & Tacrolimus \\
\hline Micafungin & $\begin{array}{l}\text { Increased serum concentration of } \\
\text { coadministered drug }\end{array}$ & Sirolimus, nifedipine, and itraconazole \\
\hline Anidulafungin & $\begin{array}{l}\text { No clinically relevant drug-drug } \\
\text { interactions }\end{array}$ & \\
\hline
\end{tabular}

NOTE: Vfend (voriconazole) package labeling: Pfizer, New York, NY; December 2007. Noxafil (posaconazole) package labeling: Schering Corporation, Kenilworth, NJ; October 2006. Cancidas (caspofungin) package labeling: Merck \& Co., Inc., Whitehouse Station, NJ; February 2005. Mycamine (micafungin) package labeling: Astellas Pharma US, Inc., Deerfield, IL; January 2008. Eraxis (anidulafungin) package labeling: Pfizer, New York, NY; May 2007.

Abbreviations: HMG-CoA, 3-hydroxy-3-methyl-glutaryl-coenzyme A; HIV, human immunodeficiency virus.

catheter-related candidemia, other hematogenously disseminated disease, or visceral involvement. ${ }^{3}$ Risk factors are present in most patients with invasive candidiasis, and include broad-spectrum antibiotics; parenteral nutrition; central catheters; hospitalization in the intensive care unit setting; renal failure; burns; gastrointestinal and cardiac surgery; and colonization with Candida, particularly at multiple sites. ${ }^{1,2}$

Historically, treatment of invasive candidiasis consisted of D-AmB, with fluconazole largely but not completely replacing amphotericin after prospective trials demonstrated comparable efficacy with markedly improved tolerability. Fluconazole has poor or uncertain activity against C. krusei and C. glabrata, however, leading to reluctance on the part of many clinicians to use it for non- $C$. albicans infection (or empirically in the unstable patient). Others have raised concerns regarding the use of fluconazole even for C. albicans in the setting of an unstable or neutropenic patient, given its fungistatic rather than fungicidal activity, although this is a theoretical rather than proven shortcoming. ${ }^{1}$ Current Infectious Diseases Society of America (IDSA) guidelines for the treatment of candidemia recommend the use of caspofungin, fluconazole, $\mathrm{D}-\mathrm{AmB}$, or the combination of $\mathrm{D}$ AmB and fluconazole. ${ }^{4}$ The IDSA recommendations are under revision, however, and we summarize newer evidence below.

Mora-Duarte et al., ${ }^{5}$ in a 2002 trial, randomized patients with invasive candidiasis to caspofungin or $\mathrm{D}-\mathrm{AmB}$, and found a favorable response in $73 \%$ and $62 \%$, respectively, which fell just short of statistical significance. Caspofungin was better tolerated than $\mathrm{D}-\mathrm{AmB}$, and the authors concluded that caspofungin was at least as effective as D$\mathrm{AmB}$, with fewer adverse effects. ${ }^{5}$ A 2007 study randomized invasive candidiasis patients to micafungin or L-AmB, and reported similar efficacy in both arms, with less drug-related adverse events in the echinocandin-treated group. ${ }^{6}$ Reboli et al. ${ }^{7}$ conducted a noninferiority trial comparing anidulafungin to fluconazole, and found a significantly 
superior outcome in the anidulafungin arm. Perhaps surprisingly, the outcome difference between the 2 groups was greater for C. albicans than for any other species. ${ }^{7}$ Although the large majority of patients in the preceding trials had candidemia, one study demonstrated a favorable response to caspofungin in $81 \%$ of patients with invasive candidal infections other than candidemia. ${ }^{8}$

Fewer data exist regarding the use of newer azoles for the treatment of invasive candidiasis. Ostrosky-Zeichner et al. $^{3}$ utilized voriconazole as salvage therapy in 52 patients with invasive candidiasis either refractory to or intolerant of other antifungals (almost all of whom had failed therapy with $\mathrm{D}-\mathrm{AmB}$ and/or other azoles), and found a $56 \%$ favorable response rate in this challenging population. More recently, Kullberg et al. ${ }^{9}$ studied voriconazole versus $\mathrm{D}$-AmB followed by fluconazole in candidemic patients, with a similar outcome but somewhat better tolerability in the voriconazole arm. We are unaware of comparative studies involving posaconazole for invasive candidiasis.

In summary, although fluconazole is the drug of choice for most invasive candidal infections, the initial use of an echinocandin should be considered when infection with a non-C. albicans species is likely, particularly if the patient is unstable. Provided the organism later proves likely to be sensitive, switching to fluconazole is reasonable, particularly given the absence of an oral echinocandin formulation. The 3 currently available echinocandins appear to be interchangeable for the treatment of serious Candida infections.

\section{NEUTROPENIC FEVER}

Neutropenia is the most critical factor leading to infection in patients with cancer. Empiric treatment with broad-spectrum antimicrobials should be initiated at the first sign of infection, since delay can lead to increased mortality. ${ }^{10}$ There are numerous causes for fever in the neutropenic host, although bacterial infection is most common. Fungal infections can cause unexplained fever and should be considered in neutropenic patients who remain febrile despite broad-spectrum antibiotics.

Fungal infections in the neutropenic host can have severe consequences. Given their high morbidity and mortality and a lack of effective diagnostic techniques for early detection, empiric antifungal therapy is mandatory in the appropriate setting. Antifungal therapy should be considered in patients who remain febrile and neutropenic for $\geq 5$ days despite broad-spectrum antibiotics. The most common fungal pathogens include Candida and Aspergillus spp. ${ }^{11}$ Other considerations include the emergence of nonalbicans Candida infections and other opportunistic pathogens such as Zygomycetes (Mucor and related pathogens), Fusarium spp, and Scedosporium spp.

Empiric antifungal coverage in the neutropenic host has evolved over the past 2 decades, with the first trials demonstrating the utility of empiric antifungal treatment in the neutropenic host published in the 1980s. These trials demonstrated that addition of D-AmB to broad spectrum antibiotics decreased development of fungal infections, and led to better outcomes. ${ }^{12,13}$ While these studies established $\mathrm{D}-\mathrm{AmB}$ as standard empiric antifungal therapy in neutropenic fever, nephrotoxicity and infusion-related reactions limited its subsequent use as less toxic alternatives were developed. The lipid formulations of amphotericin B, in particular liposomal AmB and amphotericin B lipid complex, have been shown to be as effective as D-AmB for empiric treatment of febrile neutropenia, with less toxicities but significantly higher expense. ${ }^{14,15}$ The older generation azoles itraconazole and fluconazole have also been studied. Itraconazole has been proven to be as effective as $\mathrm{D}-\mathrm{AmB}$ in febrile neutropenia with less toxicity; however, the oral capsule has erratic absorption and should be used cautiously. ${ }^{16}$

Newer agents studied for use in febrile neutropenia include caspofungin and voriconazole. Caspofungin is active against azole-resistant Candida spp and Aspergillus spp with a favorable toxicity profile, making it an attractive candidate for use in febrile neutropenia. Caspofungin was compared to L-AmB as empiric antifungal therapy in a randomized double-blind trial of 1,095 patients with febrile neutropenia. ${ }^{17}$ The overall success rate was essentially identical for both agents, demonstrating noninferiority of caspofungin therapy. Among patients with baseline fungal infections, significantly more patients receiving caspofungin than L-AmB had successful outcomes (52\% versus $26 \%$, $P=0.04)$. Overall, caspofungin was better tolerated and associated with fewer complications than L-AmB. ${ }^{17}$ The other available echinocandins, micafungin and anidulafungin, have not yet been 
studied for febrile neutropenia in randomized, controlled fashion.

Voriconazole is a second-generation azole with activity against fluconazole-resistant Candida strains; however, the minimum inhibitory concentrations (MICs) are proportionally higher, suggesting a possible cross-resistance mechanism among highly azole-resistant strains. ${ }^{18}$ Voriconazole is active against most Aspergillus spp, Fusarium spp, and Scedosporium apiospermum. ${ }^{19}$ Voriconazole was compared to L-AmB in an open-label, randomized trial of 837 patients with febrile neutropenia. ${ }^{20}$ Patients were stratified according to risk of fungal infection and previous antifungal prophylaxis. Toxic side effects were similar in both groups. Less breakthrough fungal infections were seen in the voriconazole group; however, there were more discontinuations due to lack of efficacy in patients receiving voriconazole compared to LAmB. The overall success rate was $26 \%$ with voriconazole and $31 \%$ with L-AmB $(95 \%$ confidence interval $[\mathrm{CI}]$ for absolute difference in success rates: $-10.6 \%$ to $1.6 \%$ ), with the low figures reflective not only of infection severity, but also gravity of underlying disease, persistent fever presumably not of fungal origin, and adverse drug effects. Because the predetermined definition of noninferiority for the confidence interval difference between the groups was not met, the U.S. Food and Drug Administration (FDA) voted against approval of voriconazole for febrile neutropenia.

Overall, the role of newer antifungals in the treatment of febrile neutropenia is evolving. Based on current evidence, we prefer caspofungin as the treatment of choice for patients with febrile neutropenia because of its low toxicity profile and good clinical spectrum against most likely pathogens. D-AmB has long been the gold standard; however, due to toxicity concerns, lipid-based formulations have largely replaced it, with a notable increase in cost. Voriconazole cannot be recommended at this time based on failure to meet the noninferiority endpoint when compared to L-AmB. However, for cases in which there is a high suspicion of invasive aspergillosis infection, voriconazole should be considered.

\section{INVASIVE ASPERGILLOSIS}

Invasive aspergillosis infection has become an increasing threat in immunocompromised pat- ients, including those treated for cancer, undergoing organ transplantation, or with advanced human immunodeficiency virus (HIV) infection. In particular, patients being treated for hematologic malignancies and those undergoing hematopoietic stem cell transplant (HSCT) are at highest risk, due to prolonged, severe neutropenia. Infection with invasive aspergillosis also occurs when steroids are used for treatment of graft-versushost disease in the HSCT population.

Aspergillus species are saprobic molds found ubiquitously in nature. Most diseases are caused by Aspergillus fumigatus, followed by A. flavus, A. niger, and $A$. terreus. Infection with Aspergillus can cause a wide spectrum of illnesses, ranging from allergic reactions to fulminant, lethal infections. The lungs are the most common site of primary invasive disease and are associated with high mortality, especially in severely immunocompromised patients. ${ }^{21}$ Infection is rapidly progressive and can be refractory to treatment, due to the organism's ability to grow quickly and invade blood vessels. Susceptible patients are unable to control infection and thus at high risk for dissemination and death. Prompt administration of an effective antifungal agent is necessary upon suspicion of invasive disease.

The choice of antifungals for invasive Aspergillus infection has grown significantly over the past decade. Current FDA-approved agents with activity and indications for Aspergillus infection include D-AmB and its lipid formulations, itraconazole, voriconazole, posaconazole, and caspofungin. $\mathrm{D}-\mathrm{AmB}$ and voriconazole are the only agents licensed in the US for the primary treatment of invasive aspergillosis, with $\mathrm{D}-\mathrm{AmB}$ the sole therapeutic option until recently. The lipid formulations of amphotericin B, itraconazole, and caspofungin are approved for salvage therapy. Posaconazole is licensed for prophylaxis of invasive aspergillosis in patients who are severely immunocompromised, including those with HSCT and graft-versus-host disease as well as those with hematologic malignancies and prolonged neutropenia. Besides caspofungin, the other available echinocandins, micafungin and anidulafungin, are active against Aspergillus species, but not yet FDA-approved for this indication.

Voriconazole has replaced $\mathrm{D}-\mathrm{AmB}$ as the primary treatment of invasive pulmonary aspergillosis. ${ }^{21}$ Voriconazole was compared to D-AmB in a randomized, multicenter, open-label trial of 277 
immunocompromised patients with definite or probable disease. The underlying condition in most patients was acute leukemia or allogeneic HSCT, and the majority of patients had invasive pulmonary disease. A successful outcome at week 12 was seen in $53 \%$ in the voriconazole group and $32 \%$ in the D-AmB group, with survival rates of $71 \%$ and $58 \%$, respectively; both differences were statistically significant. There were more adverse events in the D-AmB group. Overall, the authors concluded that initial therapy with voriconazole led to better responses, improved survival and fewer side effects than D-AmB. ${ }^{22}$

Caspofungin and micafungin have been studied for use as salvage therapy in invasive Aspergillus infection. Caspofungin was studied in 83 patients with invasive aspergillosis refractory to or intolerant of D-AmB, lipid formulations of amphotericin $\mathrm{B}$, or triazoles, most of whom had hematologic malignancy and allogeneic HSCT. The majority of patients had invasive pulmonary aspergillosis, and a favorable response was seen in $45 \%$ of this extremely high-risk population. ${ }^{23}$ Micafungin was evaluated in a phase II study as primary or salvage therapy for invasive aspergillosis in adults and children. Of the patients receiving micafungin alone, those receiving the drug as primary therapy had a $50 \%(\mathrm{n}=6 / 12)$ response rate, compared to $41 \%(9 / 22)$ in the salvage therapy group. ${ }^{24}$ Optimal dosing of micafungin for the treatment of Aspergillus has not yet been established.

Posaconazole, the newest triazole antifungal, has been shown to be effective for the prevention of invasive aspergillosis in immunocompromised patients $^{25,26}$ and has also been studied for the treatment of invasive disease. In an open-label trial, patients with invasive aspergillosis refractory or intolerant to conventional therapy were administered posaconazole, with historical controls as a comparator group. ${ }^{27}$ The majority of patients had underlying hematologic malignancies with approximately half undergoing HSCT, and most patients had pulmonary infection. The overall success rate was $42 \%$ for posaconazole and $26 \%$ for the control group. Posaconazole appeared to confer a survival benefit over control at 30 days and end of therapy $(P=0.0003)$.

Based on current data, we recommend voriconazole for primary treatment of invasive pulmonary aspergillosis. Alternatives include L-AmB, caspofungin, micafungin, or posaconazole; of these agents, only L-AmB has been studied as primary (as opposed to salvage) therapy for invasive aspergillosis in a reasonably-powered trial. ${ }^{28} \mathrm{We}$ agree with current IDSA guidelines, which suggest $\mathrm{L}-\mathrm{AmB}$ as a possible alternative to voriconazole for primary therapy of invasive aspergillosis in some patients, particularly where drug-drug interactions make the use of voriconazole problematic. ${ }^{21}$

\section{MUCOCUTANEOUS CANDIDIASIS}

Oropharyngeal candidiasis, or thrush, is a common infection in infants; those receiving antibiotics, chemotherapy or inhaled corticosteroids; and those with underlying immunodeficiency states. Esophageal candidiasis is most common in patients infected with HIV. Oral candidiasis usually does not cause symptoms, while esophageal disease is associated with odynophagia and dysphagia.

Candida albicans is the most common cause of mucocutaneous candidiasis. Treatment of thrush usually entails topical antifungal agents such as clotrimazole troches or nystatin, or oral azoles such as fluconazole or itraconazole. Topical therapy is ineffective for esophageal candidiasis, and oral or intravenous azoles are required as first-line therapy with fluconazole being preferred. The treatment of oral and esophageal candidiasis is often complicated by recurrence, especially in immunodeficient patients, and resistance to standard treatments occurs frequently. Identification of Candida to the species level should be performed in the setting of refractory mucocutaneous disease, as this may play a role in the choice of therapy. The 2004 IDSA Guidelines, currently under revision, contain recommendations for treatment of refractory mucocutaneous candidiasis. ${ }^{4}$ The guidelines recommend a trial of oral itraconazole for fluconazole-refractory thrush. Intravenous caspofungin and $\mathrm{D}-\mathrm{AmB}$ are usually effective alternatives. For treatment of fluconazole-refractory esophageal disease, the guidelines recommend itraconazole solution, voriconazole, or caspofungin, with $\mathrm{D}-\mathrm{AmB}$ recommended as second line therapy, though it is now seldom used in this setting due to significant adverse affects. Experience using newer antifungals is increasing, and these data are summarized below.

Voriconazole has been shown at least as effective as fluconazole in the treatment of esophageal candidiasis in immunocompromised patients. ${ }^{29} \mathrm{~A}$ 
study involving 256 patients revealed success rates of $98 \%$ for voriconazole and $95 \%$ for fluconazole. C. albicans was the most common pathogen isolated. Perfect et al. ${ }^{30}$ demonstrated the utility of voriconazole for refractory esophageal candidiasis in 38 patients. A successful outcome was seen in $61 \%$ of patients treated with intravenous followed by oral voriconazole. The most common pathogen was $C$. albicans, although the series included several cases of infection with $C$. krusei.

Caspofungin was compared to D-AmB for the treatment of esophageal candidiasis in a multicenter, double-blind, randomized trial of 128 patients. ${ }^{31}$ Caspofungin appeared to be at least as effective as D-AmB, with a significantly higher incidence of drug-related adverse effects seen in the D-AmB arm. Caspofungin was also compared to fluconazole in a double-blind, randomized trial of 177 patients with Candida esophagitis. Favorable responses were seen in $81 \%$ and $85 \%$ of caspofungin and fluconazole treated patients, respectively. A trend toward higher relapse rate 4 weeks after stopping therapy was seen with caspofungin compared to fluconazole, as was a trend toward superior eradication rates for C. glabrata in the caspofungin arm compared to the fluconazole arm, although neither reached statistical significance. $^{32}$

Micafungin was used for the treatment of esophageal candidiasis in a dose-ranging trial of 245 HIV-infected patients. ${ }^{33}$ Endoscopic combined cure rate for the $100 \mathrm{mg}$ and $150 \mathrm{mg}$ doses of micafungin $(84 \%)$ was comparable to that of intravenous fluconazole $200 \mathrm{mg} /$ day $(87 \%)$. In the posttreatment period, 9 patients in the micafungin arm had a worsening of severity score or received nonprophylactic antifungal therapy. No patients in the fluconazole group experienced a relapse.

Anidulafungin has been compared with fluconazole for the treatment of Candida esophagitis in a randomized, double-blind trial of 601 patients, with an initial endoscopic success rate approaching $100 \%$ in both groups. ${ }^{34}$ The 2 -week follow-up examination revealed that $64 \%$ and $90 \%$ of patients treated with anidulafungin and fluconazole, respectively, sustained endoscopic success $(P$ $<0.001)$.

Posaconazole was compared with fluconazole for treatment of thrush in 350 patients with HIV/ acquired immunodeficiency syndrome (AIDS) in a randomized, blinded study. ${ }^{35}$ Both posaconazole and fluconazole were administered at a dose of $200 \mathrm{mg}$ on day 1 , followed by $100 \mathrm{mg} /$ day. Clinical success occurred in $92 \%$ of patients receiving posaconazole and $93 \%$ receiving fluconazole. Mycological success was equivalent on day 14 in both arms; however, by day 42 , significantly more posaconazole recipients continued to demonstrate mycological success. Posaconazole was recently evaluated for the treatment of azole-refractory thrush and esophageal candidiasis in patients with advanced HIV infection, demonstrating a success rate of $75 \%$ in this population failing fluconazole or itraconazole therapy. ${ }^{36}$

Multiple new agents are available for the treatment of mucocutaneous candidiasis. Aside from topical antifungals for the initial treatment of thrush, fluconazole remains first line systemic therapy for both oral and esophageal candidiasis due to safety, tolerability, and cost. For fluconazole-refractory disease, newer choices include voriconazole, the echinocandins, and posaconazole. Voriconazole and posaconazole are attractive options given their oral availability. The relapse rates seen in trials with the echinocandins are concerning; however, these are useful options when azole resistance is suspected.

\section{ZYGOMYCOSIS}

Zygomycosis (often referred to less correctly as "mucormycosis") is a devastating opportunistic fungal infection that appears to be increasing in frequency. Historically, zygomycosis has commonly occurred in poorly controlled diabetic patients, particularly in the setting of diabetic ketoacidosis, and classically results in rhinocerebral disease with a relatively poor outcome. In recent years, a striking increase has been seen in patients with more profound immunosuppression, particularly those with hematologic malignancies or undergoing HSCT. Sinopulmonary rather than rhinocerebral disease is the most common manifestation in this population. ${ }^{37-39}$ Other welldescribed risk factors include iron chelation therapy with deferoxamine, intravenous drug use, solid organ transplantation, metabolic acidosis, trauma, and burns. Disease is also occasionally seen in the seemingly immunocompetent, with 176 of 929 (19\%) patients in a comprehensive review lacking an obvious risk factor. ${ }^{37,40}$

Invasive mold infections caused by the Zygomycetes are associated with a poor outcome, with 
Roden et al. ${ }^{37}$ reporting mortality in excess of $50 \%$ in their series. Mortality in patients with hematological malignancies has been reported to be particularly high. ${ }^{37,38}$ The cornerstones of successful therapy include early detection of infection, correction or improvement of immunosuppression when possible, prompt surgical debridement of infected tissue, and appropriate antifungal therapy. ${ }^{40} \mathrm{D}$-AmB has constituted standard zygomycosis therapy for decades, although it has recently been largely replaced by L-AmB. Overall survival rates have been reported to be $61 \%$ and $69 \%$ with the use of D-AmB and lipid preparations, respectively. ${ }^{37}$

Given the relatively poor outcomes and substantial infusion-related toxicity and nephrotoxicity associated with even liposomal preparations of AmB, considerable interest exists in the identification of alternative therapeutic agents. Unfortunately, echinocandins and most triazoles appear to have modest to no activity against Zygomycetes, with a recent case-control study indicating that widespread use of voriconazole in high-risk populations may be helping to drive the emergence of breakthrough zygomycosis. ${ }^{39}$ Posaconazole appears to be an exception, however; with in vitro and murine studies suggesting it compares favorably to $\mathrm{D}-\mathrm{AmB}$ in this setting. ${ }^{41-43}$ Numerous case reports describe favorable outcomes with the use of posaconazole as salvage therapy for zygomycosis, and 2 recent retrospective studies support its role in this setting. ${ }^{4,45}$ Currently, use of posaconazole for the treatment of zygomycosis is limited by the absence of an intravenous preparation, although this is reportedly under development. ${ }^{46}$ At present, the role of posaconazole in this setting appears limited to step-down therapy in those patients who have responded appropriately to L-AmB, and for salvage therapy. Although an intravenous preparation of posaconazole appears attractive as a firstline agent for zygomycosis, currently studied patients (ie, those unresponsive to or intolerant of D-AmB) may not be fully representative of a broader population, and clinical trials will be necessary before more definitive conclusions may be drawn. ${ }^{47}$

\section{ENDEMIC MYCOSES Coccidioidomycosis}

Coccidioidomycosis results from environmental exposure to either Coccidioides immitis or C. posa- dii. At least $50 \%$ of infections are asymptomatic, with the majority of the remaining individuals exhibiting acute, self-limited pulmonary symptoms. A small percentage of patients develop chronic illness, either pulmonary or disseminated disease, including involvement of skin, bone/joint, and central nervous system (CNS) ${ }^{48,49}$ Current therapy consists of either fluconazole or itraconazole for CNS disease and non-life-threatening disease elsewhere, with D-AmB reserved for pregnancy and more fulminant illness. ${ }^{49}$ Unfortunately, response failures and relapses are seen commonly with all of these agents, with a resultant need for alternative antifungals.

The echinocandins have no clear role in the treatment of coccidioidomycosis. ${ }^{49}$ More interest surrounds the use of the newer azoles, with multiple studies demonstrating excellent in vitro activity of both voriconazole and posaconazole against Coccidioides species. ${ }^{50-52}$ Several recently reported open-label studies have reported good results with the use of posaconazole for chronic coccidioidomycosis, 2 of which enrolled patients intolerant of or refractory to usual agents. ${ }^{53-55}$ Based on these data, posaconazole appears to be highly active against Coccidioides, and should perhaps be the drug of choice in the majority of patients who fail to respond to or tolerate older triazoles.

\section{Histoplasmosis}

Histoplasmosis is particularly endemic in the Ohio and Mississippi valleys, although it occurs less commonly in many other areas as well. Inhaled Histoplasma capsulatum conidia result in subclinical infection in the majority of exposed individuals, with self-limited pneumonia the rule in most others. A minority of patients will experience chronic pulmonary disease or dissemination. ${ }^{56}$ Not all disease requires treatment, with most pulmonary disease resolving spontaneously; but definite indications for treatment include moderate or severe pneumonia, chronic cavitary lung disease, CNS involvement, and progressive disseminated disease. ${ }^{56}$ Standard therapy consists of itraconazole or lipid formulations of amphotericin B, based on severity. Multiple studies have demonstrated excellent in vitro activity of voriconazole and particularly posaconazole against $H$. capsulatum. ${ }^{52,57-59}$ Recently, in 2 small series of patients, patients failing either to improve with or tolerate conventional agents demonstrated favorable out- 
comes when they were treated with voriconazole or posaconazole. ${ }^{60,61}$ Both drugs appear to be appropriate second-line agents, with posaconazole arguably preferable based on current evidence.

\section{CONCLUSIONS}

The spectrum of available antifungal agents has expanded considerably in recent years, and the advent of additional drugs is expected shortly. Well-tolerated and effective drugs are now available for most fungal infections, although the precise role for newer agents in some of these diseases has yet to be defined. Future clinical trials should help resolve these uncertainties.

Address for correspondence and reprint requests: James C. Pile, MD, Bellgreve 543, CWRU/MetroHealth Medical Center, 2500 MetroHealth Dr., Cleveland, OH 44109; Telephone: (216) 778-5162; Fax: (216) 778-4105; E-mail: james.pile@case.edu

Received 13 March 2008; revision received 1 July 2008; accepted 2 July 2008.

\section{REFERENCES}

1. Spellberg BJ, Filler SG, Edwards JE Jr. Current treatment strategies for disseminated candidiasis. Clin Infect Dis. 2006;42:244-251.

2. Bennett JE. Echinocandins for candidemia in adults without neutropenia. N Engl J Med. 2006;355:1154-1159.

3. Ostrosky-Zeichner L, Oude Lashof AML, Kullberg BJ, Rex $\mathrm{JH}$. Voriconazole salvage treatment of invasive candidiasis. Eur J Clin Microbiol Infect Dis. 2003;22:651-655.

4. Pappas PG, Rex JH, Sobel JD, et al. Guidelines for treatment of candidiasis. Clin Infect Dis. 2004;38:161-189.

5. Mora-Duarte J, Betts R, Rotstein C, et al. Comparison of caspofugin and amphotericin B for invasive candidiasis. N Engl J Med. 2002;347:2020-2029.

6. Kuse E-R, Chetchotisakd P, Arns da Cunha C, et al. Micafungin versus liposomal amphotericin B for candidaemia and invasive candidosis: a phase III randomized doubleblind trial. Lancet. 2007;369:1519-1527.

7. Reboli AC, Rotstein C, Pappas PG, et al. Anidulafungin versus fluconazole for invasive candidiasis. $N$ Engl $\mathrm{J} \mathrm{Med.}$ 2007;356:2472-2482.

8. Cornely OA, Lasso M, Betts R, et al. Caspofungin for the treatment of less common forms of invasive candidiasis. J Antimicrob Chemother. 2007;60:363-369.

9. Kullberg BJ, Sobel JD, Ruhnke M, et al. Voriconazole versus a regimen of amphotericin B followed by fluconazole for candidaemia in non-neutropenic patients: a randomized non-inferiority trial. Lancet. 2005;366:1435-1442.

10. Antoniadou A, Giamarellou H. Fever of unknown origin in febrile leucopenia. Infect Dis Clin North Am. 2007;21: 1055-1090.

11. Hughes WT, Armstrong D, Bodey GP, et al. 2002 Guidelines for the use of antimicrobial agents in neutropenic patients with cancer. Clin Infect Dis. 2002;34:730-751.
12. Pizzo PA, Robichaud KJ, Gill FA, et al. Empiric antibiotic and antifungal therapy for cancer patients with prolonged fever and granulocytopenia. Am J Med. 1982;72:101-111.

13. EORTC International Antimicrobial Therapy Cooperative Group. Empiric antifungal therapy in febrile granulocytopenic patients. Am J Med. 1989;86:668-672.

14. Walsh TJ, Finber RW, Arndt C, et al. Liposomal amphotericin B for empirical therapy in patients with persistent fever and neutropenia. $N$ Engl J Med. 1999;340: 7644-7671.

15. Prentice HG, Hann IM, Herbrecht R, et al. A randomized comparison of liposomal versus conventional amphotericin B for the treatment of pyrexia of unknown origin in neutropenic patients. Br J Haematol. 1997;98:711-718.

16. Boogaerts MA, Maertens J, Van Der Geest R, et al. Pharmacokinetics and safety of a 7 day administration of intravenous itraconazole followed by a 14-day administration of itraconazole oral solution in patients with hematologic malignancy. Antimicrob Agents Chemother. 2001;45: 981-985.

17. Walsh TJ, Teppler H, Donowitz GR, et al. Caspofungin versus liposomal amphotericin B for empirical antifungal therapy in patients with persistent fever and neutropenia. N Engl J Med. 2004;351:1392-1402.

18. Marco F, Pfaller MA, Messer S, et al. In vitro activities of voriconazole and four other antifungal agents against 394 clinical isolates of Candida spp. Antimicrob Agents Chemother. 1998;42:161-163.

19. Marco F, Pfaller MA, Messer S, et al. Antifungal activity of a new triazole, voriconazole (UK-109,496) compared with three other antifungal agents tested against clinical isolates of filamentous fungi. Med Mycol. 1998;36: 433-436.

20. Walsh TJ, Pappas P, Winston DJ, et al. Voriconazole compared with liposomal amphotericin B for empirical antifungal therapy in patients with neutropenia and persistent fever. N Engl J Med. 2002;346:225-234.

21. Walsh TJ, Anaissie EJ, Denning DW, et al. Treatment of aspergillosis: clinical practice guidelines of the Infectious Diseases Society of America. Clin Infect Dis. 2008;46: 327-360.

22. Herbrecht R, Denning DW, Patterson TF, et al. Voriconazole versus amphotericin $\mathrm{B}$ for primary therapy of invasive aspergillosis. N Engl J Med. 2002;347:408-415.

23. Maertens J, Raad I, Petrikkos G, et al. Efficacy and safety of caspofungin for treatment of invasive aspergillosis in patients refractory to or intolerant of conventional antifungal therapy. Clin Infect Dis. 2004;39:1563-1571.

24. Denning DW, Marr KA, Lau WM, et al. Micafungin (FK463), alone or in combination with other systemic antifungal agents, for the treatment of acute invasive aspergillosis. J Infect 2006;53:337-349.

25. Cornely OA, Maertens J, Winston DJ, et al. Posaconazole vs. fluconazole or itraconazole prophylaxis in patients with neutropenia. N Engl J Med. 2007;356:348-359.

26. Ullmann AJ, Lipton JH, Vesole DH, et al. Posaconazole or fluconazole for prophylaxis in severe graft versus host disease. N Engl J Med. 2007;356:335-347.

27. Walsh TJ, Raad I, Patterson TF, et al. Treatment of invasive aspergillosis with posaconazole in patients who are refractory to or intolerant of conventional therapy: an externally controlled trial. Clin Infect Dis. 2007;44:2-12. 
28. Cornely OA, Maertens J, Bresnik M, et al. Liposomal amphotericin B as initial therapy for invasive mold infection: a randomized trial comparing a high-loading dose regimen with standard dosing (AmBiLoad trial). Clin Infect Dis. 2007;44:1289-1297.

29. Ally R, Schürmann D, Dreisel W, et al. A randomized, doubleblind, double-dummy, multicenter trial of voriconazole and fluconazole in the treatment of esophageal candidiasis in immunocompromised patients. Clin Infect Dis. 2001;33: 1447-1454.

30. Perfect JR, Marr KA, Walsh TJ, et al. Voriconazole treatment for less-common, emerging, or refractory fungal infections. Clin Infect Dis. 2003;36:1122-1131.

31. Villanueva A, Arathoon EG, Gotuzzo E, et al. A randomized double-blind study of caspofungin versus amphotericin for the treatment of candidal esophagitis. Clin Infect Dis. 2001; 33:1529-1535.

32. Villanueva A, Gotuzzo E, Arathoon EG, et al. A randomized double-blind study of caspofungin versus fluconazole for the treatment of esophageal candidiasis. Am J Med. 2002; 113:294-299.

33. de Wet N, Llanos-Cuentas A, Suleiman J, et al. A randomized, double-blind, parallel-group, dose-response study of micafungin compared with fluconazole for the treatment of esophageal candidiasis in HIV-positive patients. Clin Infect Dis. 2004;39:842-849.

34. Krause DS, Simjee AE, van Rensburg C, et al. A randomized, double-blind trial of anidulafungin versus fluconazole for the treatment of esophageal candidiasis. Clin Infect Dis. 2004;39:770-775.

35. Vasquez JA, Skiest DJ, Nieto L, et al. A multicenter randomized trial evaluating posaconazole versus fluconazole for the treatment of oropharyngeal candidiasis in subjects with HIV/AIDS. Clin Infect Dis. 2006;42:1179-1186.

36. Skiest DJ, Vasquez JA, Anstead GM, et al. Posaconazole for the treatment of azole-refractory oropharyngeal and esophageal candidiasis in subjects with HIV infection. Clin Infect Dis. 2007;44:607-614.

37. Roden MM, Zaoutis TE, Buchanan WL, et al. Epidemiology and outcome of zygomycosis: a review of 929 reported cases. Clin Infect Dis. 2005;41:634-653.

38. Kontoyiannis DP, Wessel VC, Bodey GP, Rolston KVI. Zygomycosis in the 1990s in a tertiary-care cancer center. Clin Infect Dis. 2000;30:851-856.

39. Kontoyiannis DP, Lionakis MS, Lewis RE, et al. Zygomycosis in a tertiary-care center in the era of Aspergillus-active antifungal therapy: a case-control observational study of 27 recent cases. J Infect Dis. 2005;191:1350-1360.

40. Kontoyiannis DP, Lewis RE. Invasive zygomycosis: update on pathogenesis, clinical manifestations, and management. Infect Dis Clin North Am. 2006;20:581-607.

41. Sun QN, Fothergill AW, McCarthy DI, Rinaldi MG, Graybill JR. In vitro activities of posaconazole itraconazole, voriconazole, amphotericin $\mathrm{B}$, and fluconazole against 37 clinical isolates of zygomycetes. Antimicrob Agents Chemother. 2002;46:1581-1582.

42. Sun QN, Najvar LK, Bocanegra R, Loebenberg D, Graybill JR. In vivo activity of posaconazole against mucor spp. in an immunosuppressed-mouse model. Antimicrob Agents Chemother. 2002;46:2310-2312.

43. Almyroudis NG, Sutton DA, Fothergill AW, Rinaldi MG, Kusne S. In vitro susceptibilities of 217 clinical isolates of zygomycetes to conventional and new antifungal agents. Antimicrob Agents Chemother. 2007;51:2587-2590.
44. Greenburg RN, Mullane K, van Burik J-A.H, et al. Posaconazole as salvage therapy for zygomycosis. Antimicrob Agents Chemother. 2006;50:126-133.

45. van Burik J-AH, Hare RS, Solomon HF, Corrado ML, Kontoyiannis DP. Posaconazole is effective as salvage therapy in zygomycosis: a retrospective summary of 91 cases. Clin Infect Dis. 2006;42:e61-e65.

46. Malani AN, Kauffman CA. Changing epidemiology of rare mould infections. Drugs. 2007;67:1803-1812.

47. Perfect JR. Posaconazole. Drugs. 2005;65:1568-1569.

48. Galgiani JN, Ampel NM, Blair JE, et al. Coccidioidomycosis. Clin Infect Dis. 2005;41:1217-1223.

49. Saubolle MA, McKellar PP, Sussland D. Epidemiologic, clinical, and diagnostic aspects of coccidioidomycosis. J Clin Microbiol. 2007;4:26-30.

50. Gonzalez GM, Gonzalez G, Najvar LK, Graybill JR. Therapeutic efficacy of caspofungin alone and in combination with amphotericin B deoxycholate for coccidioidomycosis in a mouse model. J Antimicrob Chemother. 2007;60: 1341-1346.

51. Ramani R, Chaturvedi V. Antifungal susceptibility profiles of Coccidioides immitis and Coccidioides posadasii from endemic and non-endemic areas. Mycopathologia. 2007; 163:31-19.

52. Li R-K, Ciblak MA, Nordoff N, Pasarell L, Warnock DW, McGinnis MR. In vitro activities of voriconazole, itraconazole, and amphotericin B against Blastomyces dermatitidis, Coccidioides immitis, and Histoplasma capsulatum. Antimicrob Agents Chemother. 2000;44:1734-1736.

53. Anstead GM, Corcoran G, Lewis J, Berg D, Graybill JR. Refractory coccidioidomycosis treated with posaconazole. Clin Infect Dis. 2005;40:1770-1776.

54. Cantanzaro A, Could GA, Stevens DA, et al. Safety, tolerance, and efficacy of posaconazole therapy in patients with nonmeningeal disseminated or chronic pulmonary coccidioidomycosis. Clin Infect Dis. 2007;45:562-568.

55. Stevens DA, Rendon A, Gaona-Flores V, et al. Posaconazole therapy for chronic refractory coccidioidomycosis. Chest. 2007;132:952-958.

56. Wheat LJ, Freifeld AG, Kleiman MB, et al. Clinical practice guidelines for the management of patients with histoplasmosis: 2007 update by the Infectious Diseases Society of America. Clin Infect Dis. 2007;45:807-825.

57. Gonzalez GM, Fothergill AW, Sutton DA, Rinaldi G, Loebenberg $D$. In vitro activities of new and established triazoles against opportunistic filamentous and dimorphic fungi. Med Mycol. 2005;43:281-284.

58. Connolly P, Wheat J, Schnizlein-Bick C, et al. Comparison of a new triazole antifungal agent, Schering 56592, with itraconazole and amphotericin B for treatment of histoplasmosis in immunocompetent mice. Antimicrob Agents Chemother. 1999;439:322-328.

59. Wheat LJ, Connolly P, Smedema M, et al. Activity of newer triazoles against Histoplasma capsulatum from patients with AIDS who failed fluconazole. J Antimicrob Chemother. 2006;57:1235-1239.

60. Freifeld AG, Iwen PC, Leisak BL, Gilroy RK, Stevens RB, Kalil AC. Histoplasmosis in solid organ transplant recipients at a large midwestern university transplant center. Transpl Infect Dis. 2005;7:109-115.

61. Restrepo A, Tobin A, Clark B, et al. Salvage treatment of histoplasmosis with posaconazole. J Infect. 2007;54:319-327. 